\title{
Lipid profiles and inflammatory markers after periodontal treatment in children with congenital heart disease and at risk for atherosclerosis
}

This article was published in the following Dove Press journal:

Vascular Health and Risk Management

8 November 2013

Number of times this article has been viewed

\author{
Adriana Chassot Bresolin' \\ Mariana Menegassi \\ Pronsatti ${ }^{2}$ \\ Larissa Nicole Pasqualotto ${ }^{2}$ \\ Patricia Oehlmeyer Nassar ${ }^{3}$ \\ Alex Sandro Jorge ${ }^{4}$ \\ Edson Antonio Alves da \\ Silva ${ }^{5}$ \\ Carlos Augusto Nassar ${ }^{3}$ \\ 'Department of Biosciences and \\ Health, ${ }^{2}$ School of Dentistry, \\ ${ }^{3}$ Department of Periodontology, \\ ${ }^{4}$ School of Biochemistry, ${ }^{5} \mathrm{Graduate}$ \\ Program, Western Paraná State \\ University (UNIOESTE), Cascavel, \\ Paraná, Brazil
}

\begin{abstract}
Due to the biological associations between periodontal and cardiovascular diseases, as well as the fact that atherosclerosis begins in childhood, behavior based on oral health care and metabolic control from an early age is essential for patients with cardiovascular disease. The aim of this research was to examine the effect of full-mouth scaling and root planing on the reduction of periodontal disease in children with congenital heart disease. In this study, treatments were related to clinical periodontal parameters and also to blood ones, such as lipid profile and inflammatory markers. The patients were divided into two groups: group $1(\mathrm{n}=17)$, scaling and root planing; and group $2(\mathrm{n}=16)$, full-mouth scaling and root planing. The results showed a significant improvement in clinical periodontal parameters $(P<0.05)$ in both groups. Considering lipid parameters, total cholesterol, triglycerides, and very-low-density lipoprotein parameters showed significant improvement $(P<0.05)$. There was also an improvement in C-reactive protein (ultrasensitive) in the group treated with scaling and root planing $(P<0.05)$. Fibrinogen and interleukin-6 parameters improved $(P<0.05)$ in both groups. We suggest that both periodontal treatments were effective in children with congenital heart disease, though neither demonstrated superiority.
\end{abstract}

Keywords: periodontal disease, heart defects, congenital, lipid metabolism, inflammation mediators, children

\section{Introduction}

Congenital heart defects are currently a focus of concern for health managers who are challenged to improve quality-of-life levels and the infant-mortality rate, since cardiovascular malformations are responsible for $48 \%$ of deaths due to congenital anomalies and for $7.9 \%$ of all deaths in children under 1 year old. ${ }^{1}$ The prevalence of these heart diseases has been increasing. In adults, the prevalence of congenital heart disease has increased $85 \%$ in the last 15 years, while in children the increase has been $22 \%$ for the same period. ${ }^{2}$

Considering the importance of dental treatment in patients with congenital heart disease, there are few studies comparing dental and oral changes in this group of heart diseases. The first comprehensive study that addressed this issue was conducted by Hakala in 1967 in Helsinki University, and listed the factors that led to a higher incidence of caries and its early appearance in cyanotic children, such as the greater frequency of mouth breathing, the swelling of the gums and the high rate of gingivitis, the exceptional position of children with heart disease in the family, which assumes a protective and mistaken attitude, and dentists' aversion "for fear of complications" to treat a child with severe heart disease. ${ }^{3}$ The benefits of good oral health go beyond preventing infective endocarditis,
Correspondence: Carlos Augusto Nassar Western Paraná State University (UNIOESTE), 504/593 Rua Pernambuco, Cascavel, Paraná 85810-020, Brazil Tel +55459101 3369

Email canassar@yahoo.com 
focusing on caries prevention, and early diagnosis and treatment of periodontal disease. Moreover, children with congenital cardiac disease are more vulnerable to stressful dental procedures because of their susceptibility to periodontitis. ${ }^{4}$

Chronic periodontitis is an infection caused by Gramnegative bacteria, resulting in the destruction of tissue that supports the teeth. Some of these microorganisms inside the microbial flora of dental plaque are the main causative agents of periodontitis. These microorganisms, particularly Porphyromonas gingivalis, produce endotoxins in the form of lipopolysaccharides (LPS), generating a host-mediated immune response, which leads to tissue destruction. ${ }^{5}$ Moreover, according to the same authors, there is evidence for direct invasion by these microorganisms in cardiovascular cells.

Periodontitis is associated with an increase in C-reactive protein (CRP) and fibrinogen (FGN) levels, both of which predispose for coronary diseases. There is evidence that increased levels of systemic inflammation markers, such as CRP and interleukin (IL)-6, are also associated with cardiovascular diseases. ${ }^{6}$

On the other hand, atherosclerosis is a major public health problem, being the leading cause of morbidity and mortality. ${ }^{7}$ There is evidence that the formation of atherosclerotic plaque begins in childhood, ${ }^{8}$ corroborated by the Bogalusa study, ${ }^{9}$ which demonstrated a high association of fatty streaks in the aorta and coronary arteries with high levels of total cholesterol (TC) and low-density lipoprotein (LDL) cholesterol, in addition to a strong correlation with multiple risk factors. Therefore, it is assumed that risk-factor control in childhood may delay or attenuate the progression of atherosclerotic disease.

The prevention of periodontitis, as well as atherosclerotic disease, has generated interest in the pediatric age group, since for many years studies have shown that concentrations of LDL cholesterol are positively associated with fatty streaks in the aorta and coronary arteries of children. ${ }^{9-11}$ There is also evidence that children and adults with congenital heart disease appear to be more predisposed to these two situations, which will certainly lead to irreversible damage in the future. ${ }^{12,13}$

Health professionals must be also aware that these diseases share many risk factors and evident similarities in basic pathogenic mechanisms. ${ }^{14,15}$ Possible mechanisms for the infection-inflammation relationship of the periodontal tissues and systemic changes initiate and maintain high systemic levels of several cytokines related to the acute-phase response of inflammation. ${ }^{16,17}$ Few studies have correlated periodontal disease in children and inflammatory markers. Studying a young population between 5 and 20 years old, Shaddox et $\mathrm{al}^{18}$ observed that levels of periodontitis as a result of systemic LPS endotoxin were strongly correlated with clinical parameters and higher levels of cytokines in the gingival crevicular fluid.

More recently, an extensive review was undertaken by a working group of the American Heart Association, which concluded that "periodontal disease is associated with vascular atherosclerotic disease independent of known confounders." 19 One of the hypotheses of this connection mechanism between periodontitis and atherosclerosis would be predicted based on inflammatory mechanisms initiated by bacteria associated with periodontal lesions, locally or systemically, and then influencing the initiation or propagation of the atherosclerotic lesion. Such lesions can be initiated by inflammatory stimuli, including systemic inflammatory cytokines produced locally and chemotactic agents that cause changes in the endothelium, such as adhesion molecule upregulation. These changes promote interactions with leukocytes, which generate their migration to the intimal layer of the artery. The lipid streaks, consisting of LDL, within macrophages and dendritic cells in the intimal layer, can initiate and propagate this inflammatory response. An upregulation of the endothelium leads to the release of additional chemotactic cytokines, such as monocyte chemotactic protein 1 , which attracts monocytes or other cells and may carry bacteria into the lesion. ${ }^{20}$

Despite several studies reporting an association between periodontal disease and lipid metabolism, ${ }^{21-23}$ and other studies investigating the effect of periodontal treatment on the lipid profile, ${ }^{24-26}$ the literature does not contain any study that has investigated the periodontal treatment impact of systemically compromised patients, particularly children, because these patients may have a different response when their lipid parameters are analyzed, due to their sickness or medication.

Recently, Frisbee et $\mathrm{al}^{27}$ administered an oral health questionnaire to American children from rural communities, and the data were correlated with lipid levels and systemic inflammatory markers. These authors found a statistically significant association between indicators of poor hygiene and inappropriate preventive dental care with increased systemic inflammation markers.

The aim of this research was to investigate the effect of full-mouth scaling and root planing (FMSRP) in the reduction of periodontitis in children with congenital heart disease and correlate it with clinical periodontal parameters as well as 
levels of blood lipid and inflammatory markers in children without systemic diseases.

\section{Subjects and methods}

This was a prospective clinical study with a quantitative approach. The study was conducted in Cascavel, Paraná, Brazil, at Western Paraná State University (UNIOESTE), and was approved by the ethics committee of the university. The data-collection period was from March to December, 2012.

The 33 children selected for the study did not present any systemic involvement or commitment. As inclusion criteria, children needed to be between 7 and 12 years old with chronic periodontitis, with at least two sites with probing depth (PD) greater than $5 \mathrm{~mm}$ and clinical attachment level above $2 \mathrm{~mm}$, with bleeding on probing (BOP) and gingival inflammation on clinical examination, with the amount of plaque and calculus compatible with chronic periodontitis, ruling out the hypothesis of aggressive periodontitis. The teeth needed to be in normal position, with a minimum of 20 teeth in dental arches, with clinical examination undertaken in the vestibular, lingual/palatal, mesial, and distal surfaces. The exclusion criteria were use of antibiotics and anti-inflammatories with or without steroids in the month prior to the study, periodontal treatment in the previous 6 months, and patients with any chronic inflammatory disease.

A total of 90 children were evaluated in the dental clinic of UNIOESTE; 57 were excluded for not meeting all the inclusion criteria or because they met some exclusion criteria, or for not attending any of the stages proposed for data collection. Among the patients with congenital heart disease who agreed to participate in the study, ten presented ventricular septal defect (VSD) and one child was diagnosed with VSD + pulmonary stenosis (PS), all of them without hemodynamic repercussion (nonoperated); six presented atrial septal defect (ASD), also without repercussion, and one with ASD + PS who underwent surgical correction; three presented aortic stenosis; two presented very small patent ductus arteriosus, without surgery recommendation; two presented PS; two had undergone coarctation of the aorta surgery; one presented a congenital mitral regurgitation; and patients with cyanotic congenital heart disease had already been operated on, since three children presented tetralogy of Fallot, one had truncus arteriosus, and one had complex congenital heart disease.

Data collection consisted of three phases and was undertaken during a period of 180 days. Dental clinical evaluations were conducted at 90-day intervals: first stage (start), second stage (90 days), and third stage (180 days). Then, the labo- ratory tests were collected and measured at the initial stage and third stage.

\section{Dental clinical evaluation}

The oral examinations were performed by a single trained examiner with extensive experience in periodontics but without knowledge of the groups' composition, who through a periodontal probe with a Williams 23 determined:

1. Plaque index (PI): according to Silness and Löe, ${ }^{28}$ consists of the absence or presence of visible plaque on the tooth surface which is divided into four zones-buccal, distal, mesial, and lingual

2. Gingival index (GI): according to Löe and Silness, ${ }^{29}$ represents the dichotomization of the presence or absence of bleeding, in the same zones previously mentioned

3. PD: the distance from the bottom of the sulcus to the gingival margin, determined at six points: mesiobuccal, buccal, distobuccal, distolingual/palatal, lingual/palatal and mesiolingual/palatal from each tooth to be examined

4. Clinical attachment level (CAL): also determined at the same points of $\mathrm{PD}$

5. BOP.

\section{Laboratory evaluation}

Blood samples were obtained in the morning, after an 8-hour fast, collected in the first and third stages on the same day and prior to the dental clinical evaluation, under previous authorization by the person responsible. After collection, the blood was centrifuged at 3,200 rpm for 10 minutes and the serum stored in Eppendorf tubes. For the proinflammatory cytokine analysis, part of the serum from all patients was maintained at $-20^{\circ} \mathrm{C}$ and evaluated only after the third stage was finished. For FGN analysis, the total blood sample was collected in a tube containing sodium citrate, and the plasma was separated by centrifugation at 3,200 rpm for 10 minutes. The blood and plasma samples were obtained and analyzed to determine the lipid profile and inflammatory markers, considering the following parameters:

1. TC

2. Cholesterol fractions (LDL, very LDL [VLDL], and high-density lipoprotein [HDL])

3. Triglycerides (TGs)

4. CRP, ultrasensitive

5. FGN

6. Expression of IL-6 and tumor necrosis factor alpha (TNF- $\alpha$ ).

The levels of TC, HDL, and TGs were measured through dry-chemistry methodology by an automated biochemical 
analyzer, the Vitros ${ }^{\circledR}$ Fusion 5.1 (Johnson and Johnson, New Brunswick, NJ, USA). CRP was measured through chemiluminescent technology with the Immulite ${ }^{\circledR} 1000$ (Siemens, Munich, Germany). FGN levels were determined using the coagulometric method with the automated Sysmex CA 500 (Siemens). Inflammatory cytokines (IL-6 and TNF- $\alpha$ ) were determined by enzyme-linked immunosorbent assay (Life Technologies, Carlsbad, CA, USA), according to the protocols determined by the manufacturer, and the reading was performed with the MR-96A multicanal reader (Mindray, Shenzhen, People's Republic of China).

After the initial clinical and laboratorial evaluations, the 33 patients were divided randomly into two groups of children, who were distributed according to the treatments performed:

- Group 1 (17 children): basic periodontal treatment (conventional SRP) + mechanical control

- Group 2 (16 children) basic periodontal treatment $($ FMSRP) + mechanical control.

All patients received periodontal therapy (conventional periodontal therapy or FMSRP), oral hygiene instructions, and supra- and subgingival scaling, which were performed using Gracey curettes (Hu-Friedy, Chicago, IL, USA). The correct instructions for the mechanical controls (Bass technique + flossing) were given to each group and then repeated in all stages. The treatment for group 1 consisted of conventional periodontal SRP, and for group 2 one-stage FMSRP, which was proposed by Quirynen's group, ${ }^{30}$ consisted of disinfection of the entire oral cavity with the use of mouthwash based on chlorhexidine.

\section{Statistical analysis}

The data obtained in the dental clinical evaluation were analyzed and evaluated by analysis of variance (ANOVA; $P<0.05)$. Student's $t$-test $(P<0.05)$ was used to evaluate the lipid profile (TC, LDL, TGs, VLDL, and HDL) and FGN, since all these parameters showed a normal distribution after applying the Shapiro-Wilk test. For the assessments of CRP, IL-6, and TNF- $\alpha$, as there was not a normal distribution, the Mann-Whitney $U$ test $(P<0.05)$ was applied. For calculating the variation of the means, the data analyzed were from the first stage (initial phase) and third stage (180 days), using ANOVA $(P<0.05)$ for the data with normal distributions and the Kruskal-Wallis test $(P<0.05)$ for those without normal distributions.

\section{Results}

Of all 33 patients, $42 \%$ were male and 58\% female, and in both groups there was a predominance of girls (group 1, 59\%; group 2, 56\%). The average age was 9 years and 10 months \pm 21 months for group 1 , and 9 years and 9 months \pm 18 months for group 2 .

Regarding the periodontal treatment of these children, both treatments were shown to be effective based on clinical data presenting a significant improvement, mainly those measuring CAL and PD, with an emphasis on the 90-day period, when there was a greater reduction. In contrast, only the GI had no improvement between the 90- and 180-day periods in group 2 (FMSRP), although there was a significant improvement in this index when compared with data from the first and third stages. Because of this fact, there was a significant statistical difference in the GI when analyzing the variation of means between the SRP and FMSRP treatment groups, demonstrating superiority in the SRP group. The data obtained in the dental clinical evaluation are shown in Table 1.

The blood values related to the lipid profile (TC, LDL, HDL, TGs, VLDL) and inflammatory markers (FGN, CRP ULTRA, IL-6, TNF- $\alpha$ ) are shown in Table 2. For lipid profile, both treatments were demonstrated to be effective, with a significant improvement in almost all parameters, except LDL in the group of children who underwent SRP (group 1),

Table I Dental clinical evaluation and statistical significance

\begin{tabular}{|c|c|c|c|c|c|c|c|c|}
\hline & \multicolumn{4}{|c|}{ SRP (group I) } & \multicolumn{4}{|c|}{ FMSRP (group 2) } \\
\hline & $\begin{array}{l}\text { I st stage } \\
(0)\end{array}$ & $\begin{array}{l}\text { 2nd stage } \\
\text { ( } 90 \text { days) }\end{array}$ & $\begin{array}{l}\text { 3rd stage } \\
\text { (180 days) }\end{array}$ & $\begin{array}{l}\Delta \\
\text { (0-180 days) }\end{array}$ & $\begin{array}{l}\text { I st stage } \\
(0)\end{array}$ & $\begin{array}{l}\text { 2nd stage } \\
\text { (90 days) }\end{array}$ & $\begin{array}{l}\text { 3rd stage } \\
\text { (1 } 80 \text { days) }\end{array}$ & $\begin{array}{l}\Delta \\
\text { (0-180 days) }\end{array}$ \\
\hline PI (\%) & $46.81 \pm 0.70$ & $47.67 \pm 0.64$ & $32.65 \pm 0.53^{\#}$ & $14.16 \pm 0.75$ & $39.86 \pm 0.40$ & $26.97 \pm 0.43^{\#}$ & $22.19 \pm 0.57^{\#}$ & $17.67 \pm 1.00$ \\
\hline GI (\%) & $7.61 \pm 0.17$ & $8.36 \pm 0.17$ & $4.13 \pm 0.11^{\#}$ & $3.48 \pm 0.21$ & $5.4 I \pm 0.12$ & $3.08 \pm 0.07^{\#}$ & $4.16 \pm 0.17^{\#}$ & $1.25 \pm 0.15^{*}$ \\
\hline BOP (\%) & $20.25 \pm 0.56$ & $7.99 \pm 0.15^{\#}$ & $6.32 \pm 0.15^{\#}$ & $13.93 \pm 0.52$ & $|8.03 \pm 0.4|$ & $5.59 \pm 0.24^{\#}$ & $4.51 \pm 0.16^{\#}$ & $13.52 \pm 0.46$ \\
\hline PD (mm) & $1.88 \pm 0.01$ & $\mathrm{I} .53 \pm 0.0 \mathrm{I}^{\#}$ & $\mathrm{I} .39 \pm 0.0 \mathrm{I}^{\#}$ & $0.49 \pm 0.07$ & $1.93 \pm 0.01$ & $\mathrm{I} .50 \pm 0.0 \mathrm{I}^{\#}$ & $\mathrm{I} .43 \pm 0.0 \mathrm{I}^{\#}$ & $0.40 \pm 0.10$ \\
\hline CAL $(\mathrm{mm})$ & $1.89 \pm 0.01$ & $1.53 \pm 0.0 \mathrm{I}^{\#}$ & $\mathrm{I} .40 \pm 0.0 \mathrm{I}^{\#}$ & $0.49 \pm 0.07$ & $1.93 \pm 0.01$ & $1.50 \pm 0.0 \mathrm{I}^{\#}$ & $1.43 \pm 0.0 \mathrm{I}^{\#}$ & $0.40 \pm 0.10$ \\
\hline
\end{tabular}

Notes: \#Statistically significant difference among means within each of the treatment groups at $P<0.05$; *statistically significant difference between the variation of the means $(\Delta)$ of the two treatment groups at $P<0.05$. Data are expressed as means \pm standard error of the mean.

Abbreviations: FMSRP, full-mouth scaling and root planing; PI, plaque index; GI, gingival index; BOP, bleeding on probing; PD, probing depth; CAL, clinical attachment level; SRP, scaling and root planing. 
Table 2 Laboratory evaluation and statistical significance

\begin{tabular}{|c|c|c|c|c|c|c|}
\hline & \multicolumn{3}{|c|}{ SRP (group I) } & \multicolumn{3}{|c|}{ FMSRP (group 2) } \\
\hline & $\begin{array}{l}\text { Ist stage } \\
(0)\end{array}$ & $\begin{array}{l}\text { 3rd stage } \\
\text { (180 days) }\end{array}$ & $\begin{array}{l}\Delta \\
\text { (0-180 days) }\end{array}$ & $\begin{array}{l}\text { I st stage } \\
(0)\end{array}$ & $\begin{array}{l}\text { 3rd stage } \\
\text { (180 days) }\end{array}$ & $\begin{array}{l}\Delta \\
\text { (0-180 days) }\end{array}$ \\
\hline TC (mg/dL) & $153.76 \pm 0.60$ & $149.08 \pm 0.62^{\#}$ & $4.68 \pm 0.55$ & $|54.80 \pm 0.4|$ & $149.76 \pm 0.53^{\#}$ & $5.04 \pm 0.54$ \\
\hline LDL (mg/dL) & $85.83 \pm 0.53$ & $90.78 \pm 0.70^{\#}$ & $-4.95 \pm 0.12$ & $85.12 \pm 0.46$ & $81.92 \pm 0.62$ & $3.20 \pm 0.15^{*}$ \\
\hline $\mathrm{HDL}(\mathrm{mg} / \mathrm{dL})$ & $51.35 \pm 0.32$ & $51.57 \pm 0.23$ & $-0.22 \pm 0.04$ & $54.37 \pm 0.44$ & $53.53 \pm 0.44$ & $0.84 \pm 0.13$ \\
\hline TGs (mg/dL) & $82.94 \pm 0.86$ & $75.85 \pm 0.80^{\#}$ & $7.09 \pm 0.83$ & $76.93 \pm 0.70$ & $71.15 \pm 0.6 I^{\#}$ & $5.78 \pm 0.86$ \\
\hline VLDL (mg/dL) & $16.51 \pm 0.17$ & $15.21 \pm 0.15^{\#}$ & $1.30 \pm 0.16$ & $15.35 \pm 0.14$ & $14.30 \pm 0.1 I^{\#}$ & $1.05 \pm 0.18$ \\
\hline $\mathrm{FGN}(\mathrm{g} / \mathrm{L})$ & $2.7 I \pm 0.02$ & $2.52 \pm 0.0 \mathrm{I}^{\#}$ & $0.19 \pm 0.02$ & $2.74 \pm 0.02$ & $2.35 \pm 0.0 I^{\#}$ & $0.39 \pm 0.02$ \\
\hline CRP (mg/dL) & $0.61 \pm 0.04$ & $0.20 \pm 0.0 \mathrm{I}^{\#}$ & $0.4 I \pm 0.04$ & $0.12 \pm 0.01$ & $0.20 \pm 0.01$ & $-0.08 \pm 0.01$ \\
\hline IL-6 (pg/mL) & $1.42 \pm 0.09$ & $0.85 \pm 0.02^{\#}$ & $0.57 \pm 0.01$ & $0.92 \pm 0.02$ & $0.72 \pm 0.0 \mathrm{I}^{\#}$ & $0.20 \pm 0.02$ \\
\hline TNF- $\alpha$ (pg/mL) & $12.39 \pm 0.9$ & $15.79 \pm 1.43$ & $-3.38 \pm 0.08$ & $13.44 \pm 0.70$ & $17.73 \pm 1.30$ & $-4.29 \pm 0.13$ \\
\hline
\end{tabular}

Notes: "Statistically significant difference between means within each of the treatment groups at $P<0.05$; *statistically significant difference between the variation of the means $(\Delta)$ of the two treatment groups at $P<0.05$. Data are expressed as means \pm standard error of the mean.

Abbreviations: TC, total cholesterol; HDL, high-density lipoprotein; TGs, triglycerides; VLDL, very-low-density lipoprotein; FGN, fibrinogen; CRP, C-reactive protein; IL, interleukin; TNF, tumor necrosis factor FMSRP, full-mouth scaling and root planing; SRP, scaling and root planing; LDL, low-density lipoprotein.

which showed no improvement and remained within the desired range by age. When analyzing the variation of the means between the SRP and FMSRP treatment groups, a significant statistical difference was observed for LDL, with superiority for FMSRP.

Based on analysis of the inflammatory markers, FGN had greater prominence, with a significant improvement in both groups; however, with CRP the improvement occurred only for those who underwent SRP (group 1). For proinflammatory cytokines, IL-6 showed a significant statistical improvement for both groups. There was no statistical difference in the variation of the means between the groups when analyzing these inflammatory markers.

\section{Discussion}

Cardiovascular diseases are among those that cause death the most in contemporary society, ${ }^{31}$ despite the trend of their incidence and mortality declining, thus motivating the development of extensive studies and research aimed at improved prevention methods, diagnosis, and treatment. ${ }^{32}$ Periodontal disease has also been the subject of extensive studies and research in recent decades, representing one of the major public health problems, due to its relatively high prevalence, even in developed countries, and it is also considered the most prevalent chronic disease that affects human dentition. D'Aiuto et $\mathrm{l}^{33}$ observed that periodontitis and cardiovascular disease represent long-term chronic conditions and speculated that due to the formation of atheroma starting in childhood, periodontal treatment only in the final stage of atherosclerosis development (very late in life) may not represent an effective method of preventing cardiovascular disease progression. ${ }^{34}$ Thus, this study was conducted to seek evidence to suggest an association between periodontal and cardiovascular diseases in children with congenital heart disease.

The results of this study demonstrate that there was an improvement in the clinical periodontal parameters (Table 1) and lipid levels (TC, TGs, VLDL), both in individuals who had blood values within the normal range and those who were dyslipidemic. The periodontal treatment was effective in both groups and showed a significant improvement, as shown in Table 2, demonstrating that the control of periodontal disease can be accomplished independently of the systemic condition of the child.

Although the role of periodontitis associated with diet and behavior cannot be excluded, periodontal treatment itself was effective for lipid-profile improvement. One possible explanation for this conclusion could be the fact that the cytokines IL-6 and TNF- $\alpha$, which are present in periodontal disease, also have effects on lipid metabolism and influence the production of other cytokines, ${ }^{20,35,36}$ altering the hemodynamics and the utilization of amino acids by different tissues involved in lipid metabolism, ${ }^{37}$ or by modifying the hypothalamic-pituitary-adrenal axis and increasing the plasma concentrations of adrenocorticotropic hormones, cortisol, adrenaline, noradrenaline, and glucagon. ${ }^{38}$ Through the action of IL- $1 \beta$ and TNF- $\alpha$, exposure to microorganisms/ endotoxins results in elevated levels of free fatty acids, LDL, and TRG. These serum lipid elevations result from increased hepatic lipogenesis, ${ }^{37,38}$ adipose tissue and blood circulation lipolyses, and the TGs and LDL increased synthesis or reduced elimination, due to the decreased activity of lipoprotein lipase..$^{39,40}$ Therefore, any condition that produces IL-1 $\beta$ and TNF- $\alpha$ serum elevation (such as periodontitis) has the potential to cause hyperlipidemia. 
In this study, after evaluating the lipid profile (Table 2), the reduction of TC, TGs and VLDL levels in both groups occurred as a result of a good periodontal therapy, with a greater emphasis on treatment by FMSRP, which was corroborated by the study of $\mathrm{Oz}$ et al. ${ }^{25}$ These authors proved that periodontal treatment was beneficial for lipid metabolism.

Suggesting the effectiveness of periodontal treatment, the data analyzed in Table 2 demonstrated a significant decrease in FGN and IL-6 parameters in both groups and decreased CRP in Group 1. In this last group, there was an emphasis on SRP treatment. Vidal et $\mathrm{al}^{41}$ also noted an improvement in clinical periodontal data and a decrease in IL-6, CRP, and FGN plasma levels after periodontal treatment of hypertensive patients with severe periodontitis. IL-6 is the greatest stimulus initiator of the synthesis of acutephase proteins by hepatocytes and the first determinant in hepatic CRP production. These markers added to FGN were significantly increased in individuals who were part of the PRIME (C-reactive protein, interleukin-6, and fibrinogen as predictors of coronary heart disease) study, which showed the three of them as predictors of coronary heart disease. ${ }^{42}$ However, IL-6 was a strong and independent predictor for sudden death in this group of patients. ${ }^{43}$

In contrast to IL-6, TNF- $\alpha$ concentration was lower in patients with periodontitis than in those who had already been treated, which could be observed in both groups (Table 2). Thus, these periodontal treatments did not significantly affect the levels of TNF- $\alpha$. This was also observed by Nakajima et al, ${ }^{44}$ who proposed that it occurred due to the TNF effects having more influence on the metabolism of glucose and lipids than the inflammatory action on the endothelial cells. In this respect, the role of TNF- $\alpha$, when induced by periodontal infection, may have little atherogenic effect.

\section{Conclusion}

Considering these results, we can conclude that both periodontal treatments were effective in children with congenital heart disease, but with no superiority between the treatments. Both SRP and FMSRP have significantly improved periodontal clinical and laboratorial parameters, with an emphasis on CAL, CT, FGN, and IL-6.

\section{Acknowledgment}

This study was supported by the Foundation for PostGraduate Education (CAPES), Brasília, Brazil, and Western Paraná State University, Cascavel, Paraná, Brazil.

\section{Disclosure}

The authors report no conflicts of interest in this work.

\section{References}

1. Hagemann LL, Zielinsky P. Population screening of fetal cardiac abnormalities through prenatal echocardiography in low-risk pregnancies in the municipality of Porto Alegre. Arq Bras Cardiol. 2004;82(4): 313-326.

2. Marelli AJ, Mackie AS, Ionescu-Ittu R, Rahme E, Pilote L. Congenital heart disease in the general population: changing prevalence and age distribution. Circulation. 2007;115(2):163-172.

3. Hakala PE. Dental and oral changes in congenital heart disease. Suom Hammaslaak Toim. 1967;63(6):284-324.

4. Hallett KB, Radford DJ, Seow WK. Oral health of children with congenital cardiac diseases: a controlled study. Pediatr Dent. 1992;14(4): 224-230.

5. Buhlin K, Gustafsson A, Pockley AG, Frostegard J, Klinge B. Risk factors for cardiovascular disease in patients with periodontitis. Eur Heart J. 2003;24(23):2099-2107.

6. Packard RR, Libby P. Inflammation in atherosclerosis: from vascular biology to biomarker discovery and risk prediction. Clin Chem. 2008; 54(1):24-38.

7. World Health Organization. Global Atlas on Cardiovascular Disease Prevention and Control. Geneva: WHO; 2011.

8. Napoli C, Glass CK, Witztum JL, Deutsch R, D'Armiento FP, Palinski W. Influence of maternal hypercholesterolaemia during pregnancy on progression of early atherosclerotic lesions in childhood: Fate of Early Lesions in Children (FELIC) study. Lancet. 1999;354(9186): 1234-1241.

9. Berenson GS, Wattigney WA, Tracy RE, et al. Atherosclerosis of the aorta and coronary arteries and cardiovascular risk factors in persons aged 6 to 30 years and studied at necropsy (the Bogalusa Heart Study). Am J Cardiol. 1992;70(9):851-858.

10. McGill HC Jr, McMahan CA, Herderick EE, Malcom GT, Tracy RE, Strong JP. Origin of atherosclerosis in childhood and adolescence. Am J Clin Nutr. 2000;72(Suppl 5):1307S-1315S.

11. Modelli ME, Cherulli AS, Gandolfi L, Pratesi R. Atherosclerosis in young Brazilians suffering violent deaths: a pathological study. $B M C$ Res Notes. 2011;4:531.

12. Giannakoulas G, Dimopoulos K, Engel R, et al. Burden of coronary artery disease in adults with congenital heart disease and its relation to congenital and traditional heart risk factors. Am J Cardiol. 2009; 103(10):1445-1450.

13. Pemberton VL, McCrindle BW, Barkin S, et al. Report of the National Heart, Lung, and Blood Institute's Working Group on obesity and other cardiovascular risk factors in congenital heart disease. Circulation. 2010;121(9):1153-1159.

14. Kinane DF, Lowe GD. How periodontal disease may contribute to cardiovascular disease. Periodontol 2000. 2000;23:121-126.

15. Genco RJ, Van Dyke TE. Prevention: Reducing the risk of CVD in patients with periodontitis. Nat Rev Cardiol. 2010;7(9):479-480.

16. Ebersole JL, Cappelli D. Acute-phase reactants in infections and inflammatory diseases. Periodontol 2000. 2000;23:19-49.

17. Van Dyke TE, van Winkelhoff AJ. Infection and inflammatory mechanisms. J Clin Periodontol. 2013;40 Suppl 14:S1-S7.

18. Shaddox LM, Wiedey J, Calderon NL, et al. Local inflammatory markers and systemic endotoxin in aggressive periodontitis. J Dent Res. 2011; 90(9):1140-1144.

19. Lockhart PB, Bolger AF, Papapanou PN, et al. Periodontal disease and atherosclerotic vascular disease: does the evidence support an independent association? A scientific statement from the American Heart Association. Circulation. 2012;125(20):2520-2544.

20. Schenkein HA, Loos BG. Inflammatory mechanisms linking periodontal diseases to cardiovascular diseases. J Clin Periodontol. 2013;40 Suppl 14: S51-S69. 
21. Cutler CW, Shinedling EA, Nunn M, et al. Association between periodontitis and hyperlipidemia: cause or effect? J Periodontol. 1999; 70(12):1429-1434.

22. Katz J, Flugelman MY, Goldberg A, Heft M. Association between periodontal pockets and elevated cholesterol and low density lipoprotein cholesterol levels. J Periodontol. 2002;73(5):494-500.

23. Machado AC, Quirino MR, Nascimento LF. Relation between chronic periodontal disease and plasmatic levels of triglycerides, total cholesterol and fractions. Braz Oral Res. 2005;19(4):284-289.

24. D'Aiuto F, Nibali L, Parkar M, Suvan J, Tonetti MS. Short-term effects of intensive periodontal therapy on serum inflammatory markers and cholesterol. J Dent Res. 2005;84(3):269-273.

25. Oz SG, Fentoglu O, Kilicarslan A, et al. Beneficial effects of periodontal treatment on metabolic control of hypercholesterolemia. South Med J. 2007;100(7):686-691.

26. Pussinen PJ, Jauhiainen M, Vilkuna-Rautiainen T, et al. Periodontitis decreases the antiatherogenic potency of high density lipoprotein. J Lipid Res. 2004;45(1):139-147.

27. Frisbee SJ, Chambers CB, Frisbee JC, Goodwill AG, Crout RJ. Selfreported dental hygiene, obesity, and systemic inflammation in a pediatric rural community cohort. BMC Oral Health. 2010;10:21.

28. Silness J, Loe H. Periodontal disease in pregnancy. II. Correlation between oral hygiene and periodontal condition. Acta Odontol Scand. 1964;22:121-135.

29. Loe H, Silness J. Periodontal disease in pregnancy. I. Prevalence and severity. Acta Odontol Scand. 1963;21:533-551.

30. Quirynen M, Bollen CM, Vandekerckhove BN, Dekeyser C, Papaioannou W, Eyssen H. Full- vs partial-mouth disinfection in the treatment of periodontal infections: short-term clinical and microbiological observations. J Dent Res. 1995;74(8):1459-1467.

31. Luepker RV. Cardiovascular disease: rise, fall, and future prospects. Annu Rev Public Health. 2011;32:1-3.

32. Moura Foz A, Alexandre Romito G, Manoel Bispo C, et al. Periodontal therapy and biomarkers related to cardiovascular risk. Minerva Stomatol. 2010;59(5):271-283.

33. D’Aiuto F, Parkar M, Nibali L, Suvan J, Lessem J, Tonetti MS Periodontal infections cause changes in traditional and novel cardiovascular risk factors: results from a randomized controlled clinical trial. Am Heart J. 2006;151(5):977-984.
34. D'Aiuto F, Orlandi M, Gunsolley JC. Evidence that periodontal treatment improves biomarkers and CVD outcomes. J Clin Periodontol. 2013;40 Suppl 14:S85-S105.

35. Moldawer LL. Biology of proinflammatory cytokines and their antagonists. Crit Care Med. 1994;22(7):S3-S7.

36. Hauner H, Petruschke T, Russ M, Rohrig K, Eckel J. Effects of tumour necrosis factor alpha (TNF alpha) on glucose transport and lipid metabolism of newly-differentiated human fat cells in cell culture. Diabetologia. 1995;38(7):764-771.

37. Feingold KR, Grunfeld C. Tumor necrosis factor-alpha stimulates hepatic lipogenesis in the rat in vivo. J Clin Invest. 1987;80(1):184-190.

38. Bittencourt MSP, Figueredto CMS, Fischer RG. The influence of nonsurgical periodontal treatment over the elements of the blood, lipidic profile and glycemia of patients suffering from chronic periodontitis. Rev Cienc Med Biol. 2004;3(1):60-68.

39. Fried SK, Zechner R. Cachectin/tumor necrosis factor decreases human adipose tissue lipoprotein lipase mRNA levels, synthesis, and activity. J Lipid Res. 1989;30(12):1917-1923.

40. Moeintaghavi A, Haerian-Ardakani A, Talebi-Ardakani M, Tabatabaie I. Hyperlipidemia in patients with periodontitis. J Contemp Dent Pract. 2005;6(3):78-85.

41. Vidal F, Figueredo CM, Cordovil I, Fischer RG. Periodontal therapy reduces plasma levels of interleukin-6, C-reactive protein, and fibrinogen in patients with severe periodontitis and refractory arterial hypertension. J Periodontol. 2009;80(5):786-791.

42. Luc G, Bard JM, Juhan-Vague I, et al. C-reactive protein, interleukin-6, and fibrinogen as predictors of coronary heart disease: the PRIME study. Arterioscler Thromb Vasc Biol. 2003;23(7):1255-1261.

43. Empana JP, Jouven X, Canoui-Poitrine F, et al. C-reactive protein, interleukin 6, fibrinogen and risk of sudden death in European middle-aged men: the PRIME study. Arterioscler Thromb Vasc Biol. 2010;30(10): 2047-2052.

44. Nakajima T, Honda T, Domon H, et al. Periodontitis-associated upregulation of systemic inflammatory mediator level may increase the risk of coronary heart disease. J Periodontal Res. 2010;45(1):116-122.
Vascular Health and Risk Management

\section{Publish your work in this journal}

Vascular Health and Risk Management is an international, peerreviewed journal of therapeutics and risk management, focusing on concise rapid reporting of clinical studies on the processes involved in the maintenance of vascular health; the monitoring, prevention and treatment of vascular disease and its sequelae; and the involvement of

\section{Dovepress}

metabolic disorders, particularly diabetes. This journal is indexed on PubMed Central and MedLine. The manuscript management system is completely online and includes a very quick and fair peer-review system, which is all easy to use. Visit http://www.dovepress.com/ testimonials.php to read real quotes from published authors. 